\title{
Study of Drug Production and Quality Control Process in Factories: A Case Study in Tolid Mavade Avallieh Daroupakhsh
}

\author{
Mohammad Torkashvand ${ }^{1}$, Atefeh Hasanzadeh ${ }^{2, *}$ \\ ${ }^{1} \mathrm{MSc}$ in Chemical Engineering, Fouman Faculty of Engineering, College of Engineering, University of Tehran \\ Tehran, Iran \\ Email: torkashvand.m [AT] ut.ac.ir \\ ${ }^{2}$ Fouman Faculty of Engineering, College of Engineering, University of Tehran \\ Tehran, Iran \\ ${ }^{*}$ Email: hasanzadeh.a [AT] ut.ac.ir
}

\begin{abstract}
Tolid Mavade Avallieh Daroupakhsh Company is known as the largest producer of pharmaceutical active pharmaceutical ingredients in the Middle East and as one of the reputable companies in the field of production of narcotic and non-narcotic products worldwide.In addition to production, this complex has not neglected to consider environmental issues. In this regard, for many years, environmental protection has been seriously on its agenda, and since 2007, with the formation of an independent unit called "Production Support Unit" has followed this path more seriously. The first task of pharmaceutical manufacturers is to ensure the safety and effectiveness of their products. It is important only in the case of continuous quality control methods. For this reason the company has various departments including management, accounting, administrative and commercial affairs, planning manager, technical manager, laundry, warehouse manager, control laboratory, production control, synthesis, formulation, production management, production manager, packaging, coating, Facilities, technical management of warehouse, packaging and active pharmaceutical ingredients. Tests performed by a chemist on active pharmaceutical ingredients and drug quality are performed in a controlled laboratory. Other tasks include quality control and assurance and the development of control methods to ensure and match the production process with good GMP production operations. In the new pharmacy, another task is the responsibility of this department, and that is to ensure the compatibility of biological studies and clinical tests with good laboratory operations GLP and good clinical operations GCP. For example, these operations will be controlled by the quality control and assurance department, and their compliance with GCP, GMP, GLP is confirmed. All of these cases are discussed in detail in this paper.
\end{abstract}

Keywords-Pharmaceutical companies, quality control, Tolid Mavade Avallieh Daroupakhsh

\section{INTRODUCTION}

In some pharmaceutical companies, the tasks and operations of quality control and quality assurance are separate from each other. But either separately or together, their most important responsibilities are:

1. Testing of non-sterile products for their contamination without pathogenic germs and testing of sterile products for their sterilization.

2. Control, quality assurance, and continuous monitoring, the details of which are: proper storage of active pharmaceutical ingredients, how to synthesize drugs, preparing samples for biological laboratories, clinical tests, keeping laboratory animals, and archiving research results from clinical and biological tests in all kidneys. Laboratories Supervise the production of the final product Strict monitoring of inventory and many other operations due to the expansion and expansion of the tasks of the analytical research department and quality control and assurance of additional responsibilities to the personnel working in this department. But instead, it has created new job opportunities in the pharmaceutical industry [1].

\section{QUALITY ASSURANCE}

Temad (Tolid Mavade Avallieh Daroupakhsh) Company's Quality Assurance Unit, together with the technically responsible unit, oversees the design and proper implementation of the drug quality system, which includes optimal 
manufacturing conditions (GMP) and quality risk management in all aspects of the organization. This unit ensures that production processes are properly managed throughout the life cycle and that optimal manufacturing conditions are considered. Calibration, validation, technical inspections, and maintenance of quality systems are among the main tasks of this unit.

1. Calibration: most calibrations of equipment in terms of pressure, temperature, humidity, volume, mass, and dimensions are performed in the company's calibration laboratory and according to written instructions.

2. Validation and quality assessment: qualification of equipment and processes is the responsibility of this unit.

3. Technical inspections: Following international guidelines and Food and Drug Administration approved checklists, inspections of all company units, as well as corrective, preventive, and effectiveness checks, are performed as a team and at specified intervals. In addition, the QRM and QPR quality management system has been implemented [2].

\section{EXPERIMENTAL}

\subsection{Drugs manufactured by Temad Company}

The company's products can be divided into four categories: active pharmaceutical ingredients, semi-finished products, finished products, and intermediate products.

Category of active pharmaceutical ingredients (API) are such as:

- Acetaminophen

- Aciclovir

- Aspirin

- Calcium Atorvastatin

- Semi-finished handle such as:

- Omeprazole tablets

- Pantoprazole tablets (Figure 1)

- Esomeprazole tablets

- Lansoprazole tablets

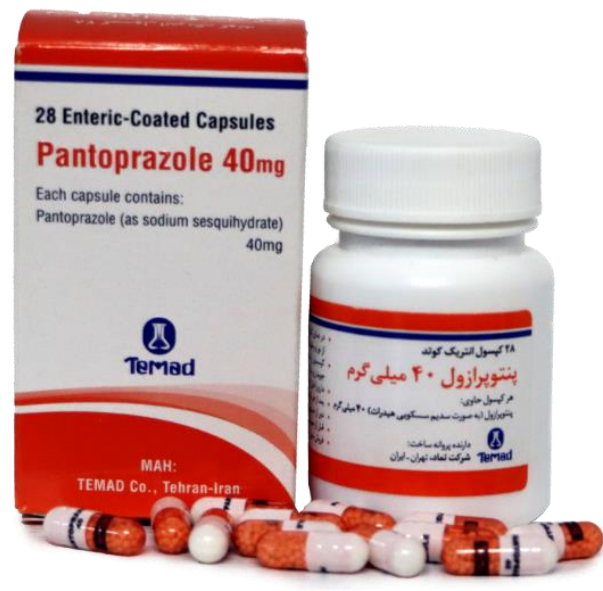

Figure 1: Pentoprazole tablets

Final product categories are such as:

- Methadone syrup

- Guaicodin Syrup

- Buprenorphine (Figure 2)

- Opium syrup 


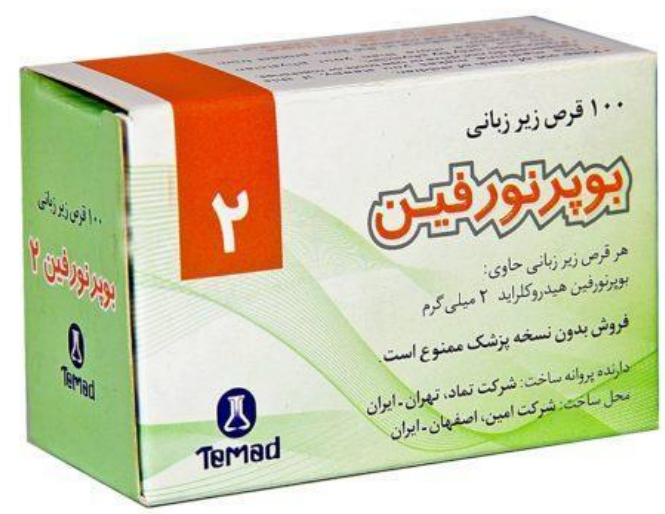

Figure 2: Buprenorphine

End product categories are such as:

- Oxymorphone base

- Noroxymorphone

\section{DRUG DESIGN AND FORMULATION}

The formulation and design of a drug product can be viewed as a step in which the formulator ensures that the right amount of drug is delivered in the right form at the right time and the right rate and that only the initial chemical properties are present [3].

The amount or quantity of the drug that can produce the desired therapeutic effects depends on many factors. In the case of official drugs, the dosage of these drugs has already been determined [4].

For certain drugs such as griseofulvin, whose better and more effective absorption depends on the particle size and specific surface area of the drug, the reduction of such a drug can increase the level of comfort food can be reduced by half or more. But it achieved the desired biological results. Since the drug is absorbed in solution; A faster dissolution can help to achieve the desired blood level and make the drug more effective [5].

\subsection{Pre-formulation studies}

The first step in developing a drug or formulation is to pay close attention to the pre-formulation data. The formulator needs to be aware of the physicochemical properties of the active ingredients before starting the formulation. The desired properties can be summarized as follows:

\subsection{Solid-state stability}

- $\quad$ against light

- temperature

- moisture

\subsection{Stability in solution}

- $\quad$ stability of drugs

- excipients

\subsection{Physicochemical properties}

Particle size density of material crystalline form compressibility melting point taste color appearance odor solubility and $\mathrm{pH}$ dissolved or suspended drug in water or other solvents. 


\subsection{Dissolution measurement}

- $\quad$ pure drug

- the effects of surfactants and by-products on drug dissolution

Drug release efficiencies are obtained according to the following formula, and by method.

Formula1:

$$
\frac{\text { The amount of drug released }}{\text { The total amount of the drug }} \times 100=\text { Drug release efficiency in percentage (\%) }
$$

The amount of drug encapsulation is calculated using the following method based on the ratio of drug loading percentage to the initial amount.

Formula2:

$$
\frac{\text { The amount of drug loaded }}{\text { The total amount of the drug }} \times 100=\text { Drug encapsulation }(\%)
$$

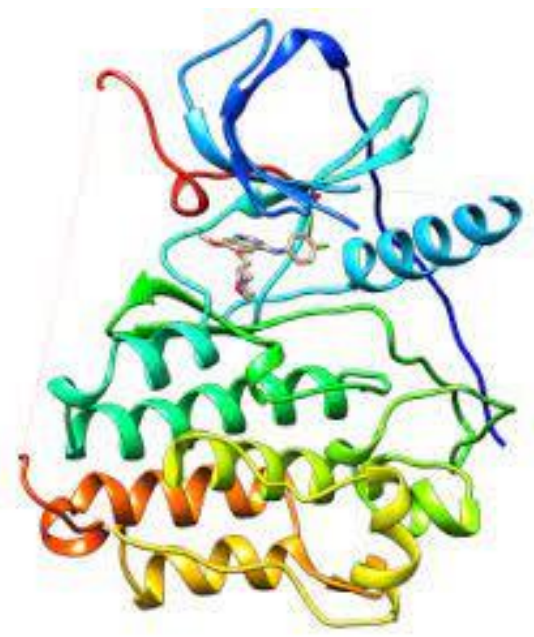

Figure 3: The early stages of drug development

\section{BASIC STEPS IN THE DESIGN OF A DRUG}

This design may include the following steps:

A. Identify the appropriate site for the release of the drug along the gastrointestinal tract.

B. Identify the appropriate manufacturing process (direct compaction of wet granulation and compaction or slag).

C. Select compatible materials for formulation.

D. Prepare experimental formulations for in vitro / in vitro laboratory and biological studies

E. In vitro testing

F. Internal tests on animals and humans

G. Develop, review, and collect as much information as possible on how to maintain the biological value and other desired properties of a new drug product.

\subsection{The desired properties in the case of a new drug}

In connection with the appropriate place for the drug to be released along the gastrointestinal tract, there are generally two types of drugs that are taken orally:

Drugs that are insoluble and are taken orally to cause local effects on the gastrointestinal tract. [6].

Drugs that are soluble and are used to create general effects after they have been dissolved and absorbed in the gut 
Each group of these drugs should be given special attention to the formulation and design of the product and its manufacturing method to create a suitable product. The effect of an insoluble drug is usually strongly dependent on the surface phenomenon, the drug design is very sensitive because the drug form must be easily formed into small particles that have large surfaces. Also, the effect of granulation formulation and density on the surface properties of materials and the ability to regenerate materials in the intestine that have suitable surface properties should be investigate [7].

In the case of a medicinal product that must be effective in general, the design of a drug that can be opened and dissolved quickly, given that the drug must be absorbed in the upper gastrointestinal tract or intestine, as well as the dissolution properties of the drug in place or before the effect of various properties must be considered. However, the drug must be designed to open and dissolve and be able to release its active ingredient at the site of absorption in the intestine [8].

\section{QUALITY CONTROL LABORATORY}

In quality management, engineering, and industrial production, the quality control, and quality engineering department is the department that is engaged in creating methods so that the factory can ensure the quality and customerfriendliness of its products. These methods and systems are usually designed in collaboration with other engineering and business disciplines [9].

The first task of pharmaceutical manufacturers is to ensure the safety and effectiveness of their products, and this is important only in the case of serious and continuous quality control methods [10].

Due to the special attention of the company's management to the principle of quality, the quality control laboratory of Temad Pharmaceutical Company, with the support of its trained and experienced personnel and having modern devices used in analysis and quantification of active pharmaceutical ingredients and products, the latest FDA quality control guidelines and international standards.

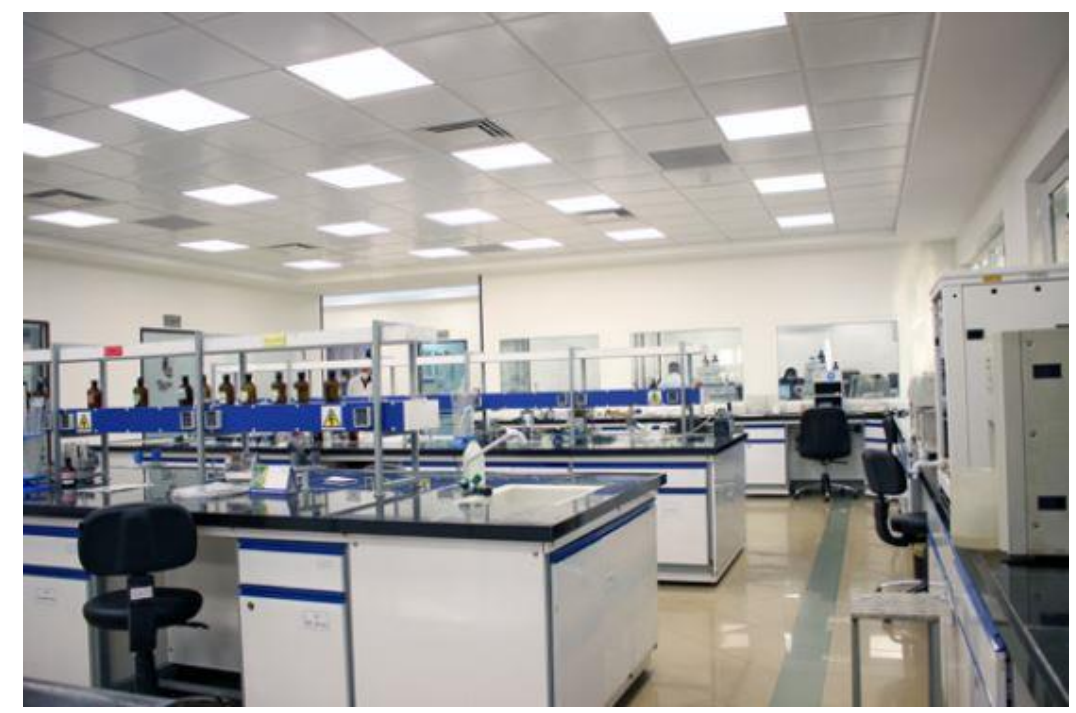

Figure 4: Quality control laboratory

In the quality evaluation of manufactured products and also in controlling the complete process of their preparation, from the purchase of active pharmaceutical ingredients to the warehousing of the final product. The laboratory has 37 staff members, most of whom are undergraduates and graduates, and operates in various departments of instrumental analysis, microbiology, IPQC, packaging control, and physicochemical control. Pharmacists, chemists, microbiologists, and all the people who work in this unit, with special attention to the safety and quality effectiveness of the products produced, try with such a sense of responsibility that the name of Temad's products is accompanied by quality.

\section{MICROBIAL CONTROL LABORATORY}

Responsibility to perform sterilization and microbial testing of products to ensure sterility and contamination of final products according to pharmacopoeial instructions, LAL(limulus amebocyte lysate) testing of products to determine febrile, microbial testing of active pharmaceutical ingredients, microbial control testing of raw water, water Distilled, 
water for washing product tanks, preparation of necessary SOPs for proper microbial controls following international drug standards and their updating, microbial control of products, active pharmaceutical ingredients, packaging containers and samples sent from the formulation, tank validation Preparations and filling devices for various production departments, air control of cleanrooms in sterile and non-sterile wards, pervasive tests, validation, endotoxin, sterilization, etc., periodic tests of products and active pharmaceutical ingredients and determination of the potency of vitamins with interest It has the latest pharmacopoeial methods [11].

\section{INSTRUMENTAL AND PHYSICOCHEMICAL LABORATORIES}

A wide range of analyzes is performed in these sections. All tests are based on valid SOPs prepared from the latest drug references, including EP (European Pharmacopoeia), BP (British Pharmacopoeia), USP (American Pharmacopoeia), and with the help of advanced devices, including chemical tests of active pharmaceutical ingredients, Performing complete tests of final production products, performing accelerated and long-term stability tests to check the conformity of the product with the desired characteristics until the expiration date set to ensure the quality of manufactured products, chemical control of distilled water produced daily to determine non-materials Permitted from the point of view of approved references, conductivity, and $\mathrm{pH}$, chemical control on the treated wastewater of the factory due to the need to comply with environmental standards, preparation of SOPs for analysis of active pharmaceutical ingredients and products following international drug standards and their updating.

\section{PRODUCTION CONTROL, CONTROL OF PACKING AND SAMPLING ITEMS}

This department has the role of supervising all parts of production (manufacturing and packaging stages). Its purpose is to implement GMP rules in all production processes (from weighing to the final packaging of the product). Supervise the operation of autoclaves, monitor the observance of GMP principles in all stages of production, monitor the quality of production equipment, control the irrigation system daily, prepare the necessary SOPs to control active pharmaceutical ingredients and products following international drug standards, and update them. Final products of all lines for delivery to the laboratory.

\section{RESULT AND DISCUSSION}

The formulation of pharmaceutical forms has undergone extensive changes in the past decades due to the precompaction of fast machines and now super-machines with automatic weight control systems as well as the possibility of access to active pharmaceutical ingredients that have direct compressibility [12].

Syrups are concentrated liquid products that contain sugar or substitutes. Syrups are one of the best food products, especially for children. In syrups, the unpleasant taste of medicinal substances is not felt due to the sweetness of the sugar as well as the high density of the liquid. Although sugar is the most widely used sweetener in syrups, other sweeteners such as dextrose, sorbitol, glycerin, saccharin, and hydroxyethyl cellulose are also used in some cases. In addition to sugar, the first three substances are eventually converted to glucose after entering the body (Glucogenic substances), while the last two substances do not have such properties. (Non-Glucogenic) [13].

\section{CONCLUSION}

In summary, it can be concluded that for the production line to reach perfect condition, pharmacists, chemists, and other quality control and quality assurance specialists will spend their efforts on important matters, including identifying important and potential issues and problems of the production line before a critical occurrence. Complete and complete product control methods Methods that are capable of determining compliance with the final product with all Pharmacopoeia and GMP requirements.

\section{ACKNOWLEDGMENT}

The authors acknowledge the partial support of this work by the staff and pharmacists of Tolid Mavade Avallieh Daroupakhsh Pharmaceutical Company for their collaborations and guidance.

\section{REFERENCES}

[1] Harbison, C.E., et al., "Scientific and Regulatory Policy Committee Points to Consider: Biological Sample Retention From Nonclinical Toxicity Studies", Toxicologic Pathology, p. 01926233211049156, 2021. 
[2] Ajit, C.P., A.M. Bhagwat, and A.P. Chaudhari, "CAPA: An important concept of Quality Assurance in Pharmaceutical Industry", Asian Journal of Research in Chemistry, vol. 14, no. 5, p. 357-362, 2021.

[3] Shaikh, R., et al., "Pharmaceutical cocrystal drug products: an outlook on product development", Trends in pharmacological sciences, vol. 39, no. 12, p. 1033-1048, 2018.

[4] Sabouhi, F., M.S. Pishvaee, and M.S. Jabalameli, "Resilient supply chain design under operational and disruption risks considering quantity discount: A case study of pharmaceutical supply chain", Computers \& Industrial Engineering, vol. 8, no. 126, p. 657-672, 2018.

[5] Cunha, S., et al., "Improving drug delivery for Alzheimer's disease through nose-to-brain delivery using nanoemulsions, Nanostructured Lipid Carriers (NLC) and in situ hydrogels", International Journal of Nanomedicine, vol. 16, p. 4373, 2021.

[6] Alqahtani, M.S., "Advances in oral drug delivery", Frontiers in Pharmacology, vol. 12, p. 62, 2021.

[7] Boyd, B.J., et al., "Successful oral delivery of poorly water-soluble drugs both depends on the intraluminal behavior of drugs and of appropriate advanced drug delivery systems", European Journal of Pharmaceutical Sciences, vol. 137: p. 104967, 2019.

[8] Karagianni, A., M. Malamatari, and K. Kachrimanis, "Pharmaceutical cocrystals: new solid phase modification approaches for the formulation of APIs", Pharmaceutics, vol. 10, no. 1, p. 18, 2018.

[9] Araújo, A.S., et al., "Key Information Related to Quality by Design (QbD) Applications in Analytical Methods Development", Braz. J. Anal. Chem, vol. 8, p. 14-28, 2021

[10] Achanta, P.S., et al., "Quantum mechanical NMR full spin analysis in pharmaceutical identity testing and quality control", Journal of Pharmaceutical and Biomedical Analysis, vol. 192: p. 113601, 2021.

[11] Dao, H., et al., "Microbial stability of pharmaceutical and cosmetic products", Aaps Pharmscitech, vol. 19, no. 1, p. 60-78, 2018.

[12] Wang, D., et al., "Selecting optimal pharmaceutical excipient formulation from life cycle assessment perspectives: A case study on ibuprofen tablet formulations", Journal of Cleaner Production, vol. 292, p. 126074, 2021.

[13] Molavi, F., M. Barzegar-Jalali, and H. Hamishehkar, "Polyester based polymeric nano and microparticles for pharmaceutical purposes: A review on formulation approaches", Journal of Controlled Release, vol. 320, p. 265-282, 2018. 